ICACCG2020 30-31 July, 2020, Ansal University, Gurgaon, India

International Journal of Technical Research \& Science (Special Issue)

\title{
MOBILE CHARGER: RELATED ISSUES AND SOLUTION
}

\author{
${ }^{1}$ Amandeep Singh, ${ }^{1}$ Arinjay Jain, ${ }^{1}$ Nagesh Singh Tomer, ${ }^{2}$ Shubahm Tiwari \\ E-Mail Id: ${ }^{1}$ aman.16ben1005@abes.ac.in, ${ }^{1}$ arinjay.16ben1013@abes.ac.in, ${ }^{1}$ nagesh.16ben1024@abes.ac.in, \\ ${ }^{2}$ shubham.tiwari@abes.ac.in \\ ${ }^{1}$ Department of Electrical and Electronics Engineering, ABES Engineering College, Ghaziabad \\ Uttar Pradesh, India \\ ${ }^{2}$ Department of Electrical and Electronics Engineering, ABES Engineering College, Ghaziabad, Uttar \\ Pradesh, India
}

\begin{abstract}
The reductions in the cost of smartphone in the last few years have ensured its reach to common people. Digital India mission has also given new dimension towards the sale of smartphones. Smartphones are now a need rather than a luxury. With the increased usage of smartphones there arises a problem related to its usage. The frequent usage invites more charge/discharge cycles of battery. The overcharging of battery reduces the life of battery. Hence, increasing the maintenance cost of device. Moreover, creates environmental issue related to the disposal of used batteries. A smart charger is devised to counter this problem. The proposed hardware not only addresses the issues related to the overcharging of battery but also provides a display of charge status through a display attached to the charger.
\end{abstract}

Keywords: Node MCU, Arduino NANO, LCD display, Relay, 7805 IC.

\section{INTRODUCTION}

Recent studies have shown that sustainability and energy reduction are the two important topics that have gained importance in the last few decades. Users want to buy products that last longer without compromising their comfort.

A current market trend shows that the market for smartphones has increased very much and will increase more in the future. Nowadays every individual is using smartphones. Children are also using smartphones so safety and the protection of smartphones are important. We all know, a battery that is used in the smartphones is made of Lithium-ion and is rechargeable.

Lithium-ion batteries now have become the power source for most of the electronic devices and are present in the market for the last two decades. It has gained importance for its advanced chemistry. It offers advantages like durability, it stores more power, charging is faster, lighter in weight, and low maintenance cost. Along with its advantages, it has disadvantages also that it causes fire or explosion due to overcharging in the worst case and life of the battery is also reduced due to overcharging [5]. It is a fact that Lithium-ion cell or battery is considered to be safe when they are operated under normal condition specified by the manufacturers and it is not safe when thermal event like smoking, fire or overcharging take place.

There are many field incidents regarding battery where the smartphones catching fire due to overcharging or heating. Although battery manufacturers provide safety regarding all this issue but keeping current injection for longer time in battery reduces the life of battery and energy is also wasted.

The table-1.1 below shows that every $0.10 \mathrm{~V}$ drop below $4.20 \mathrm{~V} /$ cell doubles the cycle but holds less capacity. Raising the voltage above $4.20 \mathrm{~V} / \mathrm{cell}$ would shorten the life of the battery [2]. So, we should not plug the phone in charging for longer duration.

Table 1.1 Discharge Cycle and capacity as a function of charge voltage limit [2]

\begin{tabular}{|l|l|l|}
\hline $\begin{array}{l}\text { Charge Level } \\
\text { (V/Cell) }\end{array}$ & Discharge Cycles & Available stored energy \\
\hline 4.30 & $150-200$ & $110-115 \%$ \\
\hline 4.25 & $200-350$ & $105-110 \%$ \\
\hline 4.20 & $300-500$ & $100 \%$ \\
\hline 4.15 & $400-700$ & $90-95 \%$ \\
\hline 4.10 & $600-1000$ & $85-90 \%$ \\
\hline 4.05 & $850-1500$ & $80-85 \%$ \\
\hline 4.00 & $1200-2000$ & $70-75 \%$ \\
\hline 3.90 & $2400-4000$ & $60-65 \%$ \\
\hline
\end{tabular}

Companies like Apple, Samsung cautions on their webpage that the capacity of Li-ion batteries reduces after each charging cycle. If you are a phone lover and buying phone after every year, than charging above 100

DOI Number: https://doi.org/10.30780/specialissue-ICACCG2020/042 pg. 55 
ICACCG2020 30-31 July, 2020, Ansal University, Gurgaon, India

International Journal of Technical Research \& Science (Special Issue) ISSN No.:2454-2024 (online) percent would not bear that much. But if you are keeping your phone for couple of years than the battery should retains its capacity for longer duration.

So in this paper we will give an idea how problems will be resolved that occur due to bad charging. Moreover we can't tell the percentage level of smartphone charged without touching it. This paper will tell the approach to display the level of charging on an external screen.

\section{BRIEF REVIEW}

In the past study was conducted over 4000 smartphone users, concluded that users mostly charge their smartphones at night and keep it charging for the whole night [1].

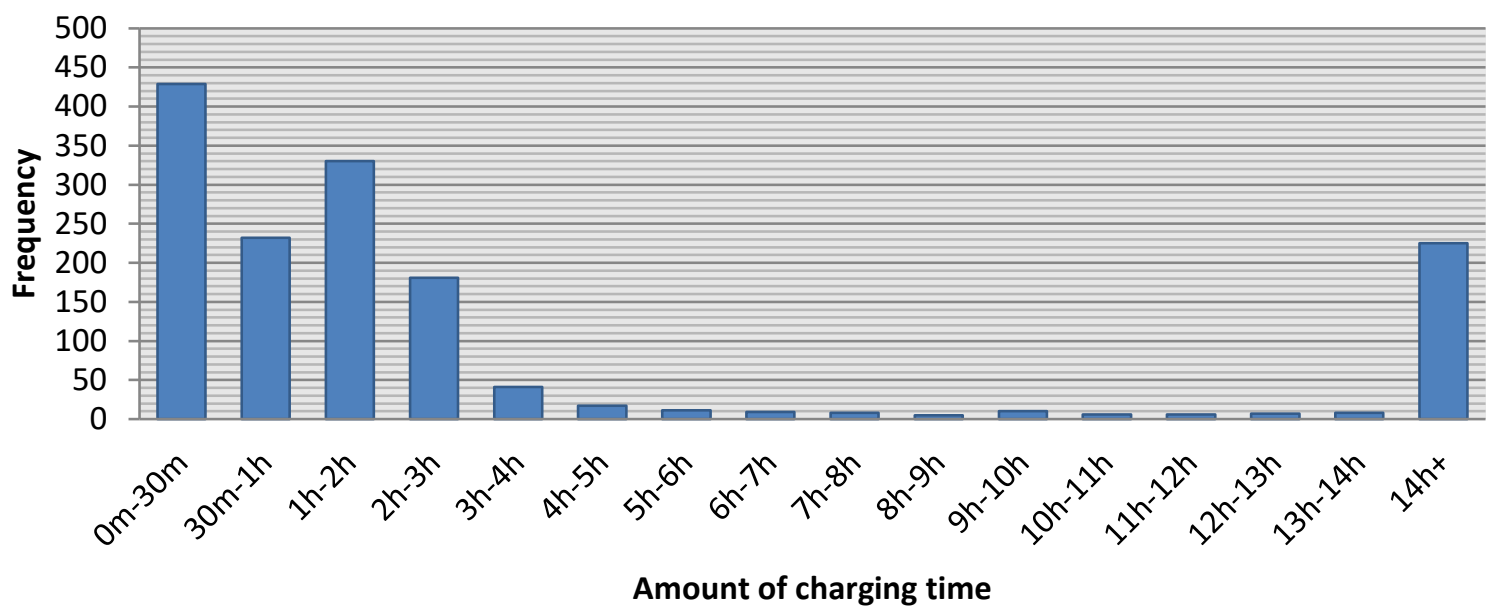

Fig. 2.1 Charging Duration (Amount of Time the Phone Remain Plugged in) [1]

The data in the graph shown in fig.2.1 has been taken from the study. Most of the charging instances occur for very short duration (left side of the graph). From graph we can also see that lot of charging instances occur during night for 14 hours or more (right Side of the graph).

People charge their phone overnight with keeping in mind that they will get $100 \%$ charged phone in the next morning. Nowadays charging the smartphones in overnight is nothing of use because it takes only 1 to 2 hours to reach 100 percent and keeping it plugged for longer time is useless.

Nearly $23 \%$ of users unplugged the phone when it is fully charged but the rest $77 \%$ plugged in for longer duration as shown in the fig.2.2. On an average, users keep the smartphones plugged for 4 hours and 39minutes after charging has been completed. This habit will definitely reduce the battery life.

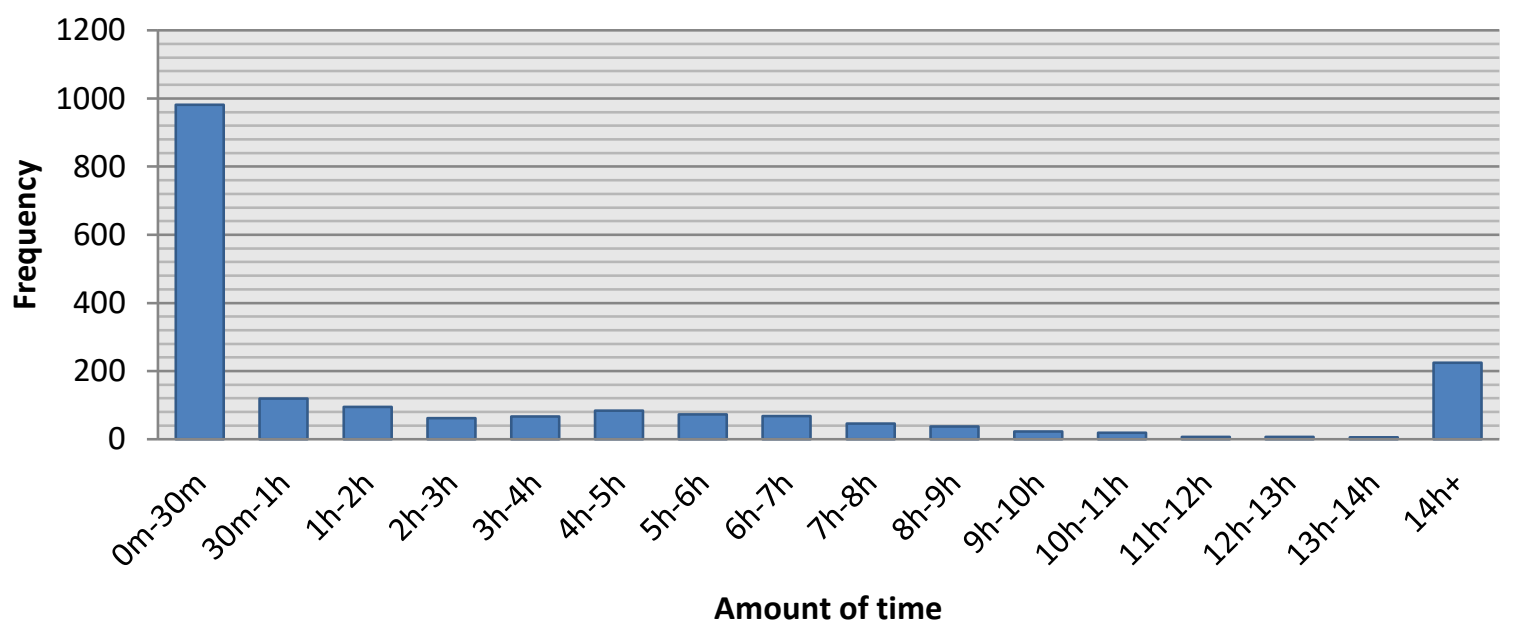

Fig. 2.2 Time Until Unplugged after the Battery is Full [1]

Most of the smartphones displays when the smartphones need to be charged but they don't notify users when to unplug when the charging is completed.

\section{PROBLEMS IN LITHIUM-ION BATTERY DUE TO IMPROPER CHARGING}

Two standards committees of the IEEE 1625 [6] and 1725 [7] have focused on the concept of safety. Li-ion battery safety should be entirety of the cell, pack, system design and the manufacturers. Various safety mechanisms are adopted during manufacturing like Positive Temperature Coefficient (PTC), Current Interrupt Device (CID) etc. to protect batteries.

Smartphones already contains chip that will protect the phones and stop charge intake. Even though charger is turnoff smartphones is continuously bounced between a full charge and a bit below a full charge. This phenomenon is called trickle charge. In this charger will top off the charge during night. Whatever charge your phones lose on its own it is charged again and maintained at 100 percent. It is highly tensed condition and if this condition is maintained for longer time battery life reduces [3].

DOI Number: https://doi.org/10.30780/specialissue-ICACCG2020/042 
At past many incidents took place in which smartphones are burned during charging as shown in the fig.3.1 below.

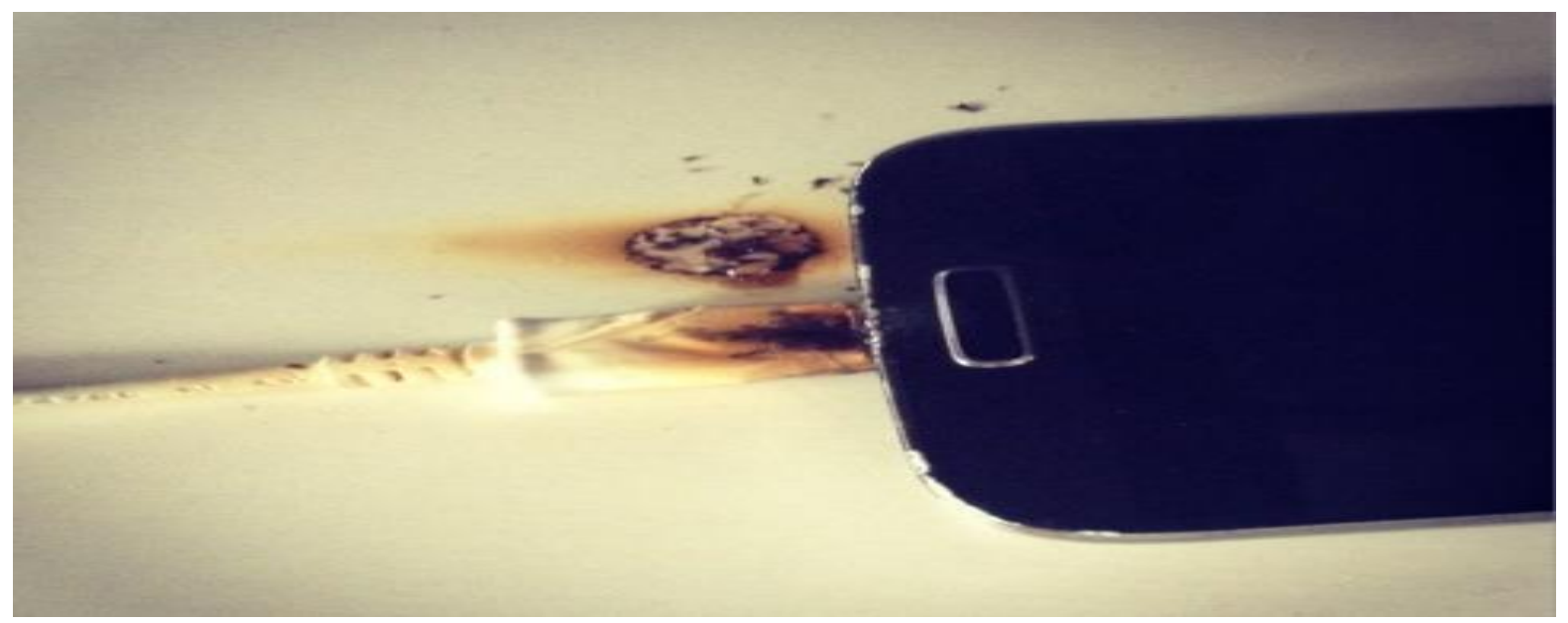

Fig. 3.1 Damaged Smartphone Due to Overcharging

\subsection{Overcharging}

Overcharging is the phenomenon in which we continuously charge the battery at a specific charge rate beyond the safe voltage limits. When the charger breaks down or we can say voltage is erroneously spotted by charging control system battery gets overcharged. When this occurs, the left over lithium ions from the cathode are removed. After this more lithium ions are injected into the anode under regular charging conditions. If the ability of lithium insertion of the carbon anode is lesser, than lithium metal is deposited in the form of dendrites on carbon and this causes an extreme reduction in thermal stability [4].

Continuous overcharging increases the temperature of the battery and reduces the life of the battery. Several exothermal reactions occur inside when temperature increase. That is why smartphones become hot when it is plugged in for longer duration.

\subsection{Thermal Runaway}

An invincible chain reaction is initiated by thermal runaway of the lithium-ion battery. Within a millisecond energy stored in the battery is released and temperature upswings quickly. The thermal runaway is initiated at a temperature of $60^{\circ} \mathrm{C}$ and becomes exceptionally severe at $100^{\circ} \mathrm{C}$. There are various factors on which thermal runaway depend. One of the root causes is overcharging in which we overcharged the battery that is specified in the data sheet. So we need a proper thermal controlling system. After the initiation of thermal runaway it becomes very difficult to stop it in an exact time, so fast and specific measurements are essential for the lithiumion battery.

Proper care is taken by the smartphones manufacturers for the problems listed above but to be more secure and to reduce fear of charging the smartphone at night and keep it plugged for longer time we have come up with an idea. In this paper we tell you the approach how we are going to make the charger smarter and how it will be helpful.

\section{PROPOSED HARDWARE FOR ELIMINATING THE CHARGING ISSUES}

We all know that charger is the only that charges the smartphone. So if we will stop the power supply of charger than charging stops. With this idea we have come up with an idea of model of charger that will stop charging the phone and cut the supply at desired level what we set and our phone is protected from continuous charging. Earlier we can't tell how much our phone is charged during charging without charging. So we have tried to solve this problem also. Explaining the model further and what components are used.

Main Components used are as follows:

\subsection{Node MCU}

In Node MCU Wi-Fi module is inbuilt. We can easily connect node MCU to the internet as compared to Arduino UNO which is more difficult to connect. Node MCU is Arduino congenial. Node MCU has Wi-Fi service available on board. With this Wi-Fi service Node MCU can connect to any Wi-Fi network as the client or it can create a network to which other Wi-Fi enabled devices can connect. Node MCU has Station (STA) mode. With the help of this we can easily connect to an existing Wi-Fi network. It can act as an HTTP server with IP address assigned by that network. Node MCU gets IP from the Wi-Fi router to which it is connected. With this IP address, it can act as an HTTP server to which any Wi-Fi device can be connected. The use of Node MCU is to receive data from the server. Data contains two parts. One part contains at what level the charger should stop charging. Second part contains the level of percentage of the mobile that need to be displayed on the screen.

\subsection{Arduino NANO}

The Arduino Nano is too small as compare to Arduino UNO. It is a complete breadboard based on the ATmega328. It has more or less the same congenial of the Arduino but in a different package. There is an absence of DC power jack, and it only works with a Mini-B USB cable rather than a standard one. It can be 
ICACCG2020 30-31 July, 2020, Ansal University, Gurgaon, India

International Journal of Technical Research \& Science (Special Issue) ISSN No.:2454-2024 (online) programmed using Arduino IDE Software. The use of Arduino in our model is to receive signal from node MCU of level of charging and to display it on the screen.

\subsection{LCD Display}

The operating voltage of the LCD module $16 * 2$ is $4.7 \mathrm{~V}$ to $5.3 \mathrm{~V}$. The current consumed without backlight is $1 \mathrm{~mA}$. It has two rows and each one can print 16 characters. It can work equally in 8 bit and 4bit mode. The display is available in green and blue backlight. Each character is built by a $5 * 8$ pixel box. It can display alphabets and numbers. On the backside of the LCD display interface IC HD44780 is attached. The only purpose of the IC is to get command and data from the MCU and route them to display significant data. LCD display will display the level of charge of the smartphone.

\subsection{Relay}

To cut off the power at 100 percent or at the desired percentage which we have selected for our smartphone to be charged we use a relay. In this proposed model 1 channel relay module is used. In electronics we use relay as a switching device. Two important factors plays an important role first is the trigger voltage and the second is load voltage and current. Trigger voltage is only essential to turn $\mathrm{ON}$ the relay that means it is only required to change the contact from NC to NO. Second factor is Load voltage and current. It is the voltage or current that $\mathrm{NC}, \mathrm{NO}$ or common terminal of the relay could tolerate.

\subsection{IC 7805}

Small amount of fluctuations is always present in the voltage source as a result fixed voltage outputs is not provided. Moreover we don't require 12V supply for Arduino NANO and node MCU so LM 7805 IC is used to give fixed voltage output range $4.7 \mathrm{~V}$ to $5.2 \mathrm{~V}$. This IC has many application areas like it can be used in reverse bias projection circuit; it is used in current regulator etc. We have used this IC to covert $12 \mathrm{~V}$ from the adapter to $5 \mathrm{~V}$ for Arduino NANO.

\section{DATA FLOW DIAGRAM}

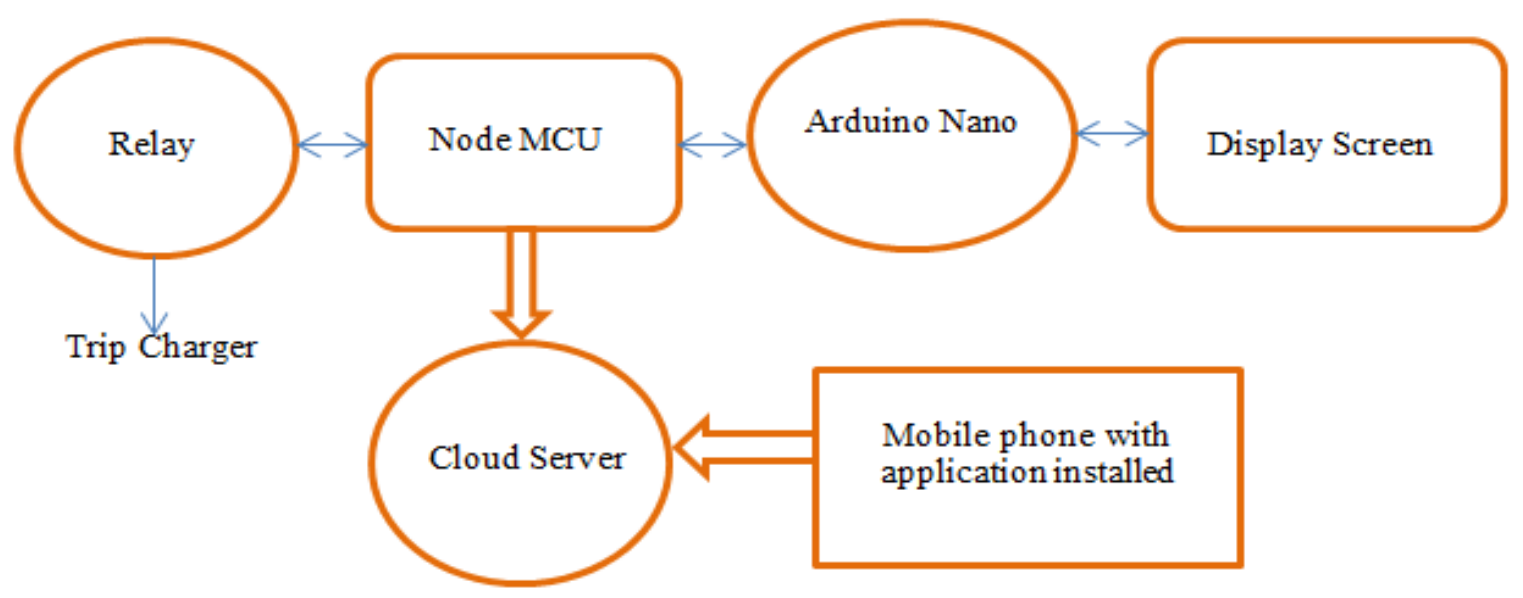

Fig. 5.1 Data Flow Diagram of the Model

The above fig.5.1 shows the direction of flow of data in our proposed model.

\subsection{Architecture explained of Proposed System}

$>$ It is very difficult to take data related to battery from the smartphone. So for this we will install an application in the smartphone. This application will send data to the server User will set the required percentage level in this application. at which the charger should be tripped during charging.

> Node MCU will access the data from the server. Node MCU will send signal to relay when desired level is reached for tripping. Node MCU will also send data to the Arduino NANO to display the level of battery in the smartphone during charging.

$>$ Relay used in the model will trip the charger.

$>$ Arduino NANO will receive the data from the Arduino NANO and display battery level during charging on the screen.

\section{CONCLUSION AND FUTURE SCOPE}

In this paper, we have explained the problems occurred due to continuous charging. For this we have tried to proposed a new hardware model which is capable of eliminating the issues in the future. It reduces the maintenance cost also. As the research and modification done on this model in future will help those users who are using smartphones for longer time and moreover with a screen option on the charger will help the users to know the battery without touching it.

\section{REFERENCES}

[1] Ferreira, D., Dey, A. K., \& Kostakos, V. (2011). Understanding Human-Smartphone.

[2] Concerns: A Study of Battery Life. Pervasive Computing, 19-33. doi:10.1007/978-3-642-21726-5_2

[3] Wu, C., Sun, J., Zhu, C., Ge, Y., \& Zhao, Y. (2015). Research on Overcharge and Over discharge Effect on

DOI Number: https://doi.org/10.30780/specialissue-ICACCG2020/042 
ICACCG2020 30-31 July, 2020, Ansal University, Gurgaon, India

International Journal of Technical Research \& Science (Special Issue) ISSN No.:2454-2024 (online)

Lithium-Ion Batteries. 2015 IEEE Vehicle Power and Propulsion Conference (VPPC). doi:10.1109/vppc.2015.7353006

[4] https://batteryuniversity.com/learn/article/how_to_prolong_lithium_based_batteries

[5] Larsson, F., \& Mellander, B.-E. (2014). Abuse by External Heating, Overcharge and Short Circuiting of Commercial Lithium-Ion Battery Cells. Journal of The Electrochemical Society, 161(10), A1611A1617. doi:10.1149/2.0311410jes

[6] C. Lampe-Onnerud et al., 14th Battery Conference on Applications and Advances, 215, 1999.

[7] IEEE 1625:2008, IEEE Standard for Rechargeable Batteries for Multi-Cell Mobile Computing Devices.

[8] IEEE 1725:2011, IEEE Standard for Rechargeable Batteries for Cellular Telephones. 